\title{
LA REVOLUCIÓN CIENTÍFICA
}

Jean Tellez*

RESUMEN: Un recorrido que comienza en 1968, cuando Morin vuelve a ser estudiante: microfísica, biología molecular, termodinámica, genética, prehistoria, cibernética, teoría de sistemas, ciencias de la información, teorías de auto-organización, epistemología, lógica matemática..., hasta llegar a entender el fenómeno humano: la suma de razón y locura.

$$
\text { yose }
$$

ABSTRACT: A journey starting in 1968 when Morin is again a student of: microphysics, molecular biology, thermodynamics, genetics, prehistory, cybernetics, systems theory, information sciences, auto-organization theory, epistemology, mathematical logic..., to finally understanding the human phenomenon: the sum of rationality and insanity.

PALABRAS CLAVE: Revolución científica, Morin, paradigma, homo sapiens, homo demens, complejidad.

KEYWORDS: Scientific Revolution, Morin, paradigm, homo sapiens, homo demens, complexity.

RECEPCIÓN: 7 de enero de 2010.

APROBACIÓN: 3 de febrero de 2010.

* Filósofo. Èditions Germina. 
CITAM Derechos Reservados.

La reproducción total o parcial de este artículo se podrá hacer si el ITAM otorga la autorización previamente por escrito. 


\section{LA REVOLUCIÓN CIENTÍFICA $^{1}$}

Elaño 1968 es decisivo: "Mayo del 68 fue para mi un nuevo comienzo". ${ }^{2}$ Una brecha se acaba de abrir en la sociedad francesa, donde "todo un reprimido, todo un inconsciente, todo un marginado, toda una necesidad, toda una libido se precipitaron". ${ }^{3}$ En Morin, "brecha" tiene un sentido muy rico. Es la falla que rompe y hace estallar lo que creíamos monolítico y estable. Pero también es la envoltura que se rompe y deja escapar su contenido, al mismo tiempo que permite derramar en su interior nuevos contenidos.

Mayo del 68 fue un retorno a las raíces. Esta revuelta de una juventud que se ahoga en las camisas de fuerza de una sociedad estancada despierta la necesidad de una revolución del adolescente consternado por Le Chemin de la vie (El camino de la vida). El hombre maduro, despertado por el adolescente interior, percibe esos acontecimientos como un apoyo para encontrarse, para nunca renunciar a sí mismo. A partir de ese momento, se desarrolla un proceso en cadena. La brecha libera todos los antiguos demonios que se van a unir en una inspiración demoníaca común: el sentido de la complejidad. Al mismo tiempo, la brecha permite una apertura donde se introducirá un apetito de saber renovado.

${ }^{1}$ Capítulo del libro La pensée tourbillonnaire. Introduction à la pensée d'Edgar Morin et Entretien avec Edgar Morin (El pensamiento turbulento. Introducción al pensamiento de Edgar Morin y Conversación con Edgar Morin), 2009, Éditions Germina. Traducción de Maiala Meza, con la colaboración de Julián Meza.

${ }^{2}$ Science avec conscience (Ciencia con consciencia), 1982, Éditions Fayard, p. 17.

${ }^{3}$ Vingt ans après (Veinte años después), en Mai 68, La Brèche (Mayo 68, La Brecha), Éditions Fayard, p. 207. 
A partir de 1968, Morin vuelve a ser estudiante. Se familiariza con una cantidad prodigiosa de saberes científicos: microfísica, biología molecular, termodinámica, genética, prehistoria, cibernética, teoría de sistemas, ciencias de la información, teorías de la auto-organización, novedades epistemológicas, lógica matemática... A partir de ese momento la búsqueda de una nueva manera de pensar es prioritaria. Esta búsqueda desembocará en una obra central: La Méthode (El Método). En cuanto al demonio de la complejidad, será el perpetuo despertar en esta nueva evolución. Está hecho de un movimiento que arremolina varias obsesiones. Sus obsesiones son, a la vez, movimientos internos irresistibles del pensamiento y de los momentos estructurantes. Sacan, sin cesar, la inteligencia de su sueño. Podemos distinguir (pero la lista no está cerrada):

El demonio de la diversidad: ver siempre la diversidad inagotable que esconde todo fenómeno que nos parece monolítico.

El demonio de la contradicción: no dejarse encerrar en un pensamiento binario que no ve más que la alternativa de lo verdadero y de lo falso. Una máxima de Niels Bohr aclara este principio: "Lo contrario de una verdad banal es un error estúpido. Lo contrario de una verdad profunda es una verdad profunda."

El demonio de la subjetividad: no olvidarse de sí mismo en un esfuerzo de conocimiento. Implicar su sensibilidad, sus pasiones, sus sueños, los acontecimientos de su vida en la necesaria reforma del pensamiento.

El demonio de la unión: luchar contra el reino de las separaciones, de las especializaciones, de los pensamientos compartimentados.

El demonio de lo desconocido: detectar la zona de sombra de todo conocimiento, el núcleo oscuro que es al mismo tiempo el núcleo poético, musical, extático.

El demonio del acontecimiento: estar siempre preparado para reformarse, para reorganizar sus ideas, para perder sus esquemas mentales en función de los acontecimientos que sobrevienen. 


\section{La revolución californiana}

En septiembre de 1969, Morin es invitado durante un año al Salk Institute for Biological Studies de San Diego, en California. ${ }^{4}$ Ese año le ofrece la ocasión de vivir en el corazón de una revolución californiana, en la que brillaban entonces sus últimos fuegos. Se sumerge completamente en la cultura hippie, la cultura de la droga, de las comunas y del rock. Los momentos fuertes de esta experiencia existencial e intelectual están relatados en Journal de Californie (Diario de California)..$^{5}$

Lo que se pone en marcha es una "revolución salvaje". No tiene un programa político claro, mezclando "fragmentos de sabiduría extrema-oriental, pólenes de hinduismo o de zen, cristianismo primitivo, comunismo primitivo, intuición de verdades arcaicas del Indio, fourierismo salvaje, marxismo vulgar, embriones de una religión de amor pancósmico..." Es una cultura compuesta por una mezcla de elementos nutritivos, que no ha tomado una forma política o ideológica determinada. Esta indeterminación constituye su carácter revolucionario. Es revolucionaria porque es adolescente y expresa las necesidades indeterminadas del adolescente. No aspira a adquirir estatus social, no está preocupada por la riqueza y la posesión, sino por la vida intensa de gasto energético, de consumación.

Al mismo tiempo que expresa los secretos del adolescente, Morin regresa al núcleo humano arcaico. "Es un neotribalismo, algo muy arcaico que surge de la punta de lanza de la modernidad". ${ }^{7}$ Es también de naturaleza biológica: es un empuje, una mutación, una desviación que parece anunciar una variante de lo humano. Sin embargo, este ascenso adolescente, ingenuo y creador, ya está amenazado: "Es demasiado débil, va a morir, es asesinado, llora..."

\footnotetext{
${ }^{4}$ Instituto de investigación creado por Jonas Salk (1914-1995), inventor de la primera vacuna contra la poliomielitis.

${ }^{5}$ Journal de Californie, 1970, Éditions du Seuil, "Points Essais” núm. 151.

${ }^{6}$ Ibid., p. 178.

${ }^{7}$ Ibid., p. 130.

${ }^{8}$ Ibid., p. 160.
} 
JEAN TELLEZ

\section{EI burbujeo cultural}

En este caldo de cultivo, las investigaciones, las reflexiones, las lecturas toman un carácter burbujeante. Morin se siente proyectado hacia la fuente interior y secreta de la existencia y de la vida: "La fuente misma de la vida se agita, está en movimiento, estructurante, desestructurante, turbulenta, en nuestro propio ser, en nuestros sueños..."9 La investigación científica y la búsqueda poética se unen en el sentimiento de alcanzar una génesis primordial, caótica y arriesgada. Una génesis espera su hora, escondida en las profundidades psíquicas: "El pensamiento, la potencia organizadora [...] buscan hacer sus combinaciones, sus asociaciones, sus instalaciones a través de mí... Espera, tantea, pisotea". ${ }^{10}$

Sueña con un pensamiento que no esté condenado a escoger entre alternativas: intelectualismo o existencialismo, empirismo o racionalismo, objetividad o subjetividad. Está atormentado por el demonio de la antropología: "Es necesario, en nombre de Dios, que me consagre a la antropología". ${ }^{11}$ Esclarecer el fenómeno humano le parece esencial. A partir de entonces es sensible a un triple fundamento de la humanidad, idea ya explorada pero no tematizada tal cual en L'Homme et la mort (El hombre y la muerte): lo humano es una realidad individual, biológica, social. Esos tres enraizamientos no deben ser estudiados por separado, sino en sus interacciones.

Morin parece cada vez más fascinado por la noción de "acontecimiento", particularmente en el sentido de una desestabilización que hace entrar en crisis dogmas y sistemas de pensamiento. Sueña que el cosmos, la vida, el mundo humano son sucesiones de acontecimientos en un sentido particularmente fuerte: azares, explosiones, metamorfosis en cadena... Está impresionado por las connotaciones y virtualidades poéticas que encierra esta idea de acontecimiento: permite tomar la espuma maravillosa, única, fugitiva de las cosas. Siente la necesidad de una antropología que vuelva a las raíces de la biología y de la cosmo-

${ }^{9}$ Ibid., p. 98.

${ }^{10}$ Ibid., p. 228.

${ }^{11}$ Ibid., p. 104. 
logía, y que pueda integrar los riesgos, la innovación, lo improbable... Está impresionado por una frase leída en Le Hasard et la nécessité (El azar y la necesidad) de Jacques Monod: "Todos los elementos son improbables". Esto lo conduce a reflexionar sobre una paradójica potencia de lo improbable: "mientras un acontecimiento es más importante, determinante, por naturaleza es más improbable". ${ }^{12}$

\section{El estudiante}

A partir de 1968, Edgar Morin vuelve a ser estudiante. La lista de libros, teorías, ideas que devora y asimila es fenomenal. Sólo se podrá rendir cuenta de ello por una lista y algunos breves comentarios (e incluso la lista estará muy incompleta). "Fue una extraordinaria confluencia de aportaciones que interactuaron las unas sobre las otras". ${ }^{13}$ A partir de entonces se encuentra atrapado en un torbellino intelectual. Todos sus centros de interés convergen en un movimiento en espiral hacia una toma de conciencia: el saber científico cambia de naturaleza a una velocidad acelerada, reclama una nueva definición del acto mismo del conocimiento.

Novedades increíbles transformaron la cara de la ciencia. Aparece un universo cada vez más apasionante. La microfísica pone de manifiesto la insuficiencia de nuestras concepciones deterministas, que ya no son válidas en la escala del átomo. Heisenberg mostró el carácter indeterminado de los fenómenos crepusculares: una partícula puede ser descrita a la vez como un cuerpo individualizado y como una onda (mientras que estas dos descripciones son contradictorias). La vieja idea de sustancia se hunde. A partir de entonces, la materia tiene cara de enigma. Por su lado, el cosmos adquiere una dimensión profundamente temporal (mientras que durante muchos siglos fue pensado como inmutable): surge de un Big-Bang inicial y se incrementa a una velocidad vertiginosa en las explosiones de estrellas y los choques de galaxias.

${ }^{12}$ Ibid., p. 56.

${ }^{13}$ Mes démons (Mis demonios), p. 247. 
Antes de irse a California, empieza a participar en las reuniones del "Grupo de los diez", bajo la égida de Jacques Robin. Este último reúne en su casa a intelectuales, científicos de diferentes disciplinas, particularmente biólogos. Morin descubre la cibernética. Esta "ciencia de las máquinas" cautiva los espíritus. Vemos la posibilidad de elucidar la naturaleza de los seres vivos, parecidos a máquinas auto reguladas. El poder explicativo de la cibernética promete extenderse a otros dominios: la sociología, las ciencias del comportamiento animal y humano.

En California empieza el gran festín intelectual que ya no se interrumpirá. Morin empieza a iniciarse en las teorías de la información de Claude Shannon, Warren Weaver, Léon Brillouin; en la cibernética de Norbert Wiener, de William Ross Ashby y Grégory Bateson; en la teoría general de sistemas de Ludwig von Bertalanffy. También descubre la importancia del problema ecológico. En esa época la conciencia ecológica es casi inexistente en Francia: el famoso "informe Meadows", Limits to Growth, aparecerá en $1972 .{ }^{14}$

Entiende bastante rápido la necesidad de sobrepasar "las tres teorías" (cibernética, teoría de la información, teoría de sistemas) e ir hacia una teoría de la auto-organización. Henri Atlan lo inicia en la teoría de los autómata auto reproductores de John von Neumann, así como en las problemáticas de la auto-organización y del "orden a partir del ruido" de Heinz von Foerster. Asimila las ideas de auto referencia y de autopoiesis de los chilenos Francisco Varela y Humberto Maturana. También llaman su atención los trabajos de Ilya Prigogine sobre las estructuras disipativas, los fenómenos organizados espontáneos que se mantienen en ciertas condiciones de inestabilidad.

Se interesa de cerca en la termodinámica. Las reflexiones de Erwin Schrödinger sobre el segundo principio de termodinámica lo cautivan. Ese principio establece que el desorden crece de manera irreversible en las transformaciones físicas. Desde un punto de vista filosófico, eso quiere decir que el desgaste, la degradación, la disipación, son el destino fatal de toda estructura organizada. En What is life? (1944) Schrödinger señala que los seres vivos, que mantienen y acrecientan su organización, constituyen una excepción del segundo principio.

${ }^{14}$ En francés será publicado en 1973. 
Se vuelve hacia los trabajos de matemática de Kurt Gödel y de Alfred Tarski. Gödel demostró que todo sistema lógico que puede generar la aritmética contiene proposiciones que no pueden ser demostradas a partir de los propios axiomas de ese sistema. Es un poco técnico, pero sugiere que hay un límite en el poder de demostración de las matemáticas. Lógica y matemáticas, disciplinas de la certidumbre absoluta, ¡incluso ellas conocen su crisis en el siglo XX!

Lee a los epistemólogos Imre Lakatos y Paul Feyerabend. En Thomas Kuhn encuentra la idea de paradigma. Un paradigma es un consenso adoptado por la comunidad científica dominante, un poco a la manera como se adopta una visión general del mundo. En el interior de este consenso los científicos formulan sus problemas, escogen sus experiencias, definen la frontera entre lo que es ciencia y lo que no lo es. De esta manera, en el corazón de la investigación científica reina lo no científico: jesquemas mentales, concepciones filosóficas, inercias, miedos a los replanteamientos! Morin también lee la obra de Karl Popper, La lógica del descubrimiento científico. Popper se interesa en el criterio que le permite asegurar que una teoría es científica. Demuestra que este criterio es la "falsabilidad": ¡la teoría misma debe ofrecer la posibilidad de ser refutada! ¡Toda teoría que afirma su verdad no es científica! ¡Todas las construcciones teóricas son provisionales!

Al mismo tiempo, "descubre el admirable texto de Husserl sobre la crisis de la ciencia europea". Este texto, escrito durante los años treinta, denuncia el acercamiento reductor de las ciencias: los científicos sólo se interesan en un mundo de hechos objetivos y descuidan los seres concretos y vivos, en particular su propia naturaleza viva. Preconizando una "objetividad rigurosa", se desinteresan en su subjetividad. Su enfoque está entonces incompleto, ciego, en gran medida es irreflexivo. La ausencia de atención en "el enigma de la subjetividad" crea una situación de profunda oscuridad que explica, según Husserl, esta crisis de la ciencia europea. 
JEAN TELLEZ

\section{La complejidad humana}

Le Paradigme perdu (El paradigma perdido), primera obra importante de este período, es un ensayo de antropología. ${ }^{15}$ El proyecto de arraigamiento de la antropología en la biología se pone en práctica. El "paradigma perdido" es la naturaleza humana que se volvió inexpugnable a causa de la compartimentación de la antropología y de la biología. Esta obra testimonia también la amplificación y la diversificación de lecturas y de centros de interés. Morin reflexiona sobre los datos más recientes de la prehistoria, así como sobre los estudios consagrados a los grupos de monos en libertad.

El rechazo al corte entre ciencias humanas y ciencias de la naturaleza encuentra su justificación desde las primeras páginas del libro: todos los rasgos que creemos específicamente humanos, la organización social, la técnica, el lenguaje, la afectividad ya están presentes en los monos. La separación radical hombre/mono y cultura/naturaleza no tiene entonces razón de ser. Es necesario ver al hombre en el mono y al mono en el hombre, la cultura en la naturaleza y la naturaleza en la cultura. Esto proporciona una forma de método para determinar los rasgos humanos fundamentales: los comportamientos más característicos que observamos en nuestro primos primates son ya humanidad. Tenemos ahí las bases genéticas de la humanidad.

Los grupos de monos son ordenados en clanes y jerarquías, pero constantemente soportan riesgos que introducen desorden: luchas entre los machos adultos, individuos con comportamientos marginales, integración de los jóvenes. Lejos de ser desestabilizados, utilizan los desórdenes y los conflictos para organizarse y volverse a organizar permanentemente. Estas perturbaciones ponen a la organización social en peligro, llevándola permanentemente al borde de la desintegración.

De esta manera comprendemos lo que es una relación "compleja": es la asociación de dos tendencias que se oponen y, al mismo tiempo, se refuerzan mutuamente. Las relaciones que tejen una sociedad son un

${ }^{15}$ Le Paradigme perdu: la nature humaine (El paradigma perdido: la naturaleza humana), 1973, Éditions du Seuil, "Points Essais”, núm. 109. 
ejemplo: "una sociedad se autoproduce sin cesar porque se autodestruye sin cesar". ${ }^{16}$ Es la ocasión para señalar el papel esencial de los grupos de jóvenes. En los grupos de monos, los machos adultos están separados en general. Pero los jóvenes, por medio de los juegos y las curiosidades, introducen innovaciones que el conjunto del grupo es susceptible de adoptar.

\section{EI rejuvenecimiento}

Los trabajos del anatomista holandés Louis Bolk (1866-1930) sobre el fenómeno de "fetalización" o de "neotenia" impresionaron a Morin en la época de Arguments (Argumentos). Bolk señala que el humano nace con rasgos del feto: gran cerebro, ausencia de vellos, arco de la ceja. La especie humana sólo produce prematuros: para sobrevivir necesitan una larga maduración en el seno de la sociedad.

De esta manera, el humano es un ser de indeterminación y de plasticidad. Dispone de un reserva perpetua de juventud. Toda nuestra vida guardamos una capacidad de aprender, de cambiar, de transformarnos. La juventud es, entonces, el demonio que nos despierta de la torpeza intelectual y existencial. Es un fuego, nunca completamente extinguido, de una infancia interior. Ninguna vida está nunca cerrada; la metamorfosis, la aventura son siempre posibles: "estos adultos endurecidos en sus determinaciones aún llevan dentro un Mowgli mal dormido, listo para volver a empezar una experiencia, una educación". ${ }^{17}$

De ahí la importancia, en toda sociedad, de los jóvenes y de los adolescentes. Son seres indeterminados, no han adoptado una personalidad fija, no han paralizado sus potencialidades en actividades sociales especializadas. De hecho, la infancia se prolonga mucho tiempo en los humanos. Los comportamientos infantiles y adolescentes, de juego, de curiosidad, persisten en los adultos, incluso en los ancianos. Esta característica se explica por el tiempo considerable que se necesita para adaptarse a una cultura cada vez más compleja: técnicas cada

${ }^{16}$ Ibid., p. 50.

${ }^{17}$ L'Homme et la mort (El hombre y la muerte), p. 97. 
JEAN TELLEZ

vez más sofisticadas, adquisición del lenguaje. Pero lo inverso es cierto: la prolongación de la infancia favorece la adquisición de una cultura cada vez más compleja. La cultura humana supone una infancia interminable y la infancia interminable del humano enriquece sus potencialidades de cultura.

\section{EI torbellino cerebral}

El rejuvenecimiento se puede poner en paralelo con la cerebralización, es decir, el increíble desarrollo del cerebro. La complejidad del cerebro es tal que sólo pudo ser posible por un proceso de engendramiento turbulento. La ya muy rica cultura de los primeros homínidos estimuló el desarrollo del cerebro; ese desarrollo enriqueció a su vez la cultura, que estimuló de nuevo el desarrollo cerebral.

Pero es sólo un aspecto de la complejidad del proceso. Encontramos aquí una dialéctica de tres términos: rejuvenecimiento, cerebralización, cultura. El rejuvenecimiento estimula la adquisición cultural, la cual estimula la cerebralización, la cual estimula la adquisición cultural, la cual... Notemos que cada una de estas instancias se desarrolla sobre un fondo de desorden y con un riesgo permanente de desorganización. El cerebro es un fantástico desorden donde miles de millones de neuronas establecen, con un gran componente de azar, miles de millones de millones de conexiones. Por su lado, la cultura se enriquece de elementos que permanentemente amenazan con desorganizarla: riesgos, innovaciones, sueños... El comportamiento juvenil es en sí mismo errático y fantasioso. De esta manera la organización humana se caracteriza por un muy alto grado de complejidad: una "hiper-complejidad".

\section{Sapiens demens}

La sapiencia de homo sapiens nace de un ruido de fondo caótico de sueños, perturbaciones mentales, fantasmas, alucinaciones. Increíblemente, esto es una "sapiencia": capacidades de pensar, de ordenar, 
de verificar, se estabilizan en ese maelstrom mental. Pero esto hace del homo sapiens un homo sapiens-demens (sabio-loco). La hominización es entonces doble. Por una parte, con la racionalidad técnica y lógica, el hombre desarrolla una toma de conciencia siempre más rica que real. Por otro lado, desarrolla una misma capacidad para dejarse invadir por los mitos y las posesiones. La racionalidad puede ser puesta al servicio de las mitificaciones y de las posesiones, que es el terreno mismo de la locura.

De esta manera, Morin reanuda con los clásicos, que incluían la locura en la definición del hombre. "Los hombres, decía Pascal, están tan necesariamente locos que sería estar loco, con otro tipo de locura, no estar loco." Estos mismos clásicos estaban conscientes de las relaciones sutiles entre locura y razón. Montaigne: “¿De qué se hace la más sutil locura, sino de la más sutil sabiduría?" Pascal: "Dos excesos: excluir la razón, sólo admitir la razón.” Para Morin, el hombre desarrolla desorden mental: fantasma, delirio, alucinaciones, posesión, así como desarrolla facultades de orden: racionalidad, sociabilidad, ética, política. La locura, que amenaza con destruir la sapiencia del Homo sapiens, se convierte también en lo que la alimenta y la refuerza. Por su lado, esta sapiencia, que puede curar la locura, siempre corre el riesgo de reforzarla. Si queremos entender el fenómeno humano es necesario que aprendamos a unir, sin perder nada de su antagonismo, las nociones de razón y locura. 
CITAM Derechos Reservados.

La reproducción total o parcial de este artículo se podrá hacer si el ITAM otorga la autorización previamente por escrito. 